\title{
El uso excesivo de pantallas está asociado con labilidad emocional en niños preescolares
} Excessive screen time is associated with emotional lability in

a. Departamento de Pediatría, Facultad de Medicina de Afyonkarahisar Sağlık Bilimleri Üniversitesi, Afyonkarahisar, Turquía.

b. Departamento de Pediatría, Facultad de Medicina de Mersin Üniversitesi, Mersin, Turquía.

c. Departamento de Pediatría, Facultad de Medicina de Hacettepe Üniversitesi, Ankara, Turquía.

d. Departamento de Pediatría, Hospital Pediátrico Dr. Sami Ulus, Ankara, Turquía.

e. Departamento de Salud Infantil y Adolescente, Ministerio de Salud, Ankara, Turquía.

f. Departamento de Psiquiatría Infantil y Adolescente, Facultad de Medicina de Hacettepe Üniversitesi, Ankara, Turquía.

g. Departamento de Pediatría, Hospital Etimesgut Sehit Sait Ertürk, Ankara, Turquía.

h. Departamento de Pediatría, Yıldırım Beyazıt Üniversitesi, Hospital de Investigación Yenimahalle, Ankara, Turquía.

Correspondencia:

Dra. Ayse Oflu: ayseoflu@gmail.com

Financiamiento: Ninguno.

Conflicto de intereses: Ninguno que declarar.

Recibido: 23-5-2020

Aceptado: $11-9-2020$

\section{preschool children}

\author{
Dra. Ayse Oflu, Profesora ayudante ${ }^{a}$ Dra. Ozlem Tezol, Profesora ayudante ${ }^{b}$, \\ Dra. Songul Yalcin, PhD y Profesorac, Dra. Deniz Yildiz d Dra. Nilgun Caylan ${ }^{e}$, \\ Dra. Dilsad F. Ozdemir, Profesoraf, Dra. Seyma Cicek y Dra. Meryem E. Nergiz ${ }^{h}$
}

\section{RESUMEN}

Introducción. En estudios anteriores, el uso excesivo o la exposición temprana a pantallas se asoció con atención deficiente, falta de control de la conducta, retraso del lenguaje y déficit en la función ejecutiva. El objetivo de este estudio fue investigar la relación entre el tiempo de uso de pantallas y la regulación emocional, que afecta las relaciones sociales de los niños de 2 a 5 años. Población y métodos. Estudio descriptivo transversal en un hospital universitario del $1 .^{\circ}$ de enero al $1 .^{\circ}$ de marzo de 2018. Se incluyó a madres de niños sanos de 2 a 5 años con un uso de pantallas inferior a 1 hora o superior a 4 horas. A quienes aceptaron participar se les administró una encuesta estructurada y la Emotion Regulation Checklist para padres.

Resultados. De los 240 niños participantes, 98 (el $40,8 \%$ ) tenían un uso de pantallas $\geq 4$ horas. Ser cuidado por la madre, tener 12 meses o más durante la primera exposición y no estar acompañado por los padres al usarlas se asociaron con $\geq 4$ horas de uso de pantallas ( $p=0,002 ; p=0,002 ; p=0,012$, respectivamente). La proporción de participantes con una puntuación alta de labilidad/negatividad $(\mathrm{L} / \mathrm{N})$ fue significativamente mayor entre los niños con $\geq 4$ horas de uso de pantallas y que no estaban acompañados por sus padres al usarlas $(p=0,004$; $p=0,033$, respectivamente).

Conclusiones. Este estudio determinó que un uso excesivo de pantallas se asocia con labilidad emocional durante esta etapa temprana de la infancia.

Palabras clave: regulación emocional, uso de pantallas, niño.

http:/ / dx.doi.org/10.5546/aap.2021.106

Texto completo en inglés:

http:/ / dx.doi.org/10.5546/ aap.2021.eng.106

Cómo citar: Oflu A, Tezol O, Yalcin S, Yildiz D, et al. El uso excesivo de pantallas está asociado con labilidad emocional en niños preescolares. Arch Argent Pediatr 2021;119(2):106-113.

\section{INTRODUCCIÓN}

En los últimos años, hubo un aumento notable en la diversidad y el uso de los medios de comunicación. Los medios tradicionales (la televisión) y nuevos (los teléfonos celulares, los teléfonos inteligentes, las tabletas y las redes sociales) pasaron a dominar la vida y el tiempo libre de muchos niños. ${ }^{1}$ Esta diversidad y este aumento en el uso de los medios de comunicación también afectó a los niños en edad preescolar. Hoy en día, para los niños preescolares, el uso de pantallas no solo implica ver televisión, sino también usar computadoras y dispositivos móviles con pantalla táctil. ${ }^{2}$ Según las recomendaciones de la Academia Estadounidense de Pediatría, los niños menores de 2 años no deben estar expuestos a pantallas, y un uso de pantallas de 1 hora o más al día se considera excesivo para los niños de entre 2 y 5 años. Además, se debe limitar el uso de los medios digitales en los niños durante las comidas o una hora antes de acostarse, para que puedan crecer y desarrollarse correctamente. ${ }^{3}$ Recientemente, la Academia Estadounidense de Pediatría ha publicado nuevas recomendaciones para los hábitos de consumo de medios durante la pandemia de COVID-19, donde se establece una rutina diaria estructurada para los niños y los adolescentes y se limita el uso de pantallas para entretenimiento. Durante la crisis de la COVID-19, se suele permitir un mayor uso de pantallas para entretenimiento. Sin 
embargo, también se recomienda que estas no ocupen la mayor parte de las horas de vigilia de los niños ni interfieran en el sueño. ${ }^{4}$

Los resultados de investigaciones previas sobre el efecto que tiene el tiempo de uso de pantallas en los procesos cognitivos varían en los niños preescolares. El uso excesivo o la exposición temprana a pantallas se asocia con atención deficiente, falta de control de la conducta, retraso del lenguaje y déficit en la función ejecutiva. ${ }^{5}$ También se ha informado que el uso excesivo de pantallas produce cambios neuroanatómicos relacionados con una menor empatía y un control de los impulsos y un procesamiento emocional deficientes. ${ }^{6}$ En algunos estudios, se observó que el tiempo de uso de pantallas no es un factor de predicción sólido de problemas en estas habilidades cognitivas. ${ }^{7}$ Se informó que, a partir de los 2 años, los programas de televisión bien planificados y adecuados a la edad, con objetivos educativos específicos, pueden ser una fuente adicional para el temprano desarrollo del lenguaje y de la alfabetización de los niños. Los programas de calidad también promueven aspectos relacionados con el desarrollo cognitivo, como las actitudes raciales positivas y el juego creativo. ${ }^{8}$

La regulación emocional (RE) se define como los procesos extrínsecos e intrínsecos responsables de controlar, evaluar y modificar las reacciones emocionales. La RE comprende la gestión y la organización de varios sistemas y componentes, como los sistemas internos y componentes sociales y conductuales. ${ }^{9} \mathrm{La}$ RE ofrece comportamientos estratégicos más adaptativos y fomenta respuestas adecuadas y flexibles en diferentes contextos emocionales. ${ }^{10}$ La RE se desarrolla gradualmente a lo largo del tiempo, con una serie de habilidades adquiridas tras el nacimiento. ${ }^{11}$ Este proceso de desarrollo supone un desarrollo neurobiológico (desarrollo del lóbulo frontal), un desarrollo conceptual (comprensión de los procesos emocionales) y habilidades de socialización. Se trata de un proceso muy importante, ya que, a partir de este, se desarrollan las diferencias individuales en las habilidades de regulación emocional de los adultos. Los niños interactúan más con el mundo exterior, y los contextos sociales se amplían en la etapa preescolar. Durante este período, los niños adquieren más habilidades de regulación emocional a medida que se dan cuenta de que tienen la posibilidad de acercarse, alejarse o cambiar las emociones que pueden atraer la atención emocional. ${ }^{12}$

Teniendo presente la importancia de las interacciones sociales para adquirir la habilidad de la RE, el objetivo de este estudio fue evaluar las asociaciones entre el tiempo de uso de pantallas y la RE de los niños de entre 2 y 5 años.

\section{MATERIALES Y MÉTODOS \\ Diseño del estudio}

Se trató de un estudio descriptivo transversal realizado en un hospital universitario entre el $1 .^{\circ}$ de enero de 2018 y el $1 .^{\circ}$ de marzo de 2018. Se incluyó en el estudio a las madres de niños sanos de entre 2 y 5 años con un tiempo de uso de pantallas inferior a 1 hora o superior a 4 horas. Se excluyó a los niños con antecedentes de prematuridad y peso insuficiente al nacer, a quienes no habían sido amamantados y a quienes tenían un retraso en el desarrollo del lenguaje y enfermedades físicas y psiquiátricas crónicas. Se determinaron estos criterios de exclusión para evitar el efecto de ciertas características que podrían ocasionar problemas conductuales. A las madres que aceptaron participar se les administró una encuesta estructurada y el cuestionario Emotion Regulation Checklist (ERC) (Lista de verificación de la regulación emocional) para padres.

El comité de ética local otorgó la autorización para llevar a cabo el estudio. Todos los procedimientos del estudio se realizaron de acuerdo con la Declaración de Helsinki.

\section{Recolección de datos}

En función de los registros de meses anteriores, se calculó que 1570 madres acudían a los consultorios pediátricos externos cada mes. En un estudio multicéntrico realizado anteriormente en Turquía, se observó que la proporción de niños con un uso de pantallas superior a 4 horas era del 19,7\%.13 Según estos datos, el tamaño mínimo de la muestra se calculó en 226 con un intervalo de confianza del $95 \%$ con la calculadora de OpenEpi (https: // www.openepi.com/SampleSize/SSPropor. Htm) a partir de la siguiente ecuación: $\mathrm{n}=$ $\left[\operatorname{DEFF}{ }^{*} \mathrm{~Np}(1-\mathrm{p})\right] /\left[\left(\mathrm{d}^{2} / \mathrm{Z}^{2}{ }_{1-\alpha / 2}^{*}(\mathrm{~N}-1)+\mathrm{p}^{*}(1-\mathrm{p})\right]\right.$. Los parámetros se establecieron como tamaño de la población $(\mathrm{N}): 3140, \%$ de frecuencia de $(\mathrm{p})$ : 19,7\% +/-5, límites de confianza como $\%(\mathrm{~d}): 5 \%$.

Al igual que en otros estudios, ${ }^{14}$ solo se incluyó a parejas de madre e hijo. El sesgo potencial sería mínimo, ya que es posible obtener información más confiable y precisa sobre los niños por 
parte de las madres. En segundo lugar, en esta región, la mayoría de los niños acudían al hospital acompañados por sus madres. Dado que el hospital donde se realizó el estudio es una institución de salud a la que acuden personas de todos los niveles socioculturales, se determinó que la muestra era suficientemente representativa. Se informó a las parejas de madres e hijos que estaban en la sala de espera antes de la consulta acerca del contenido y el objetivo del estudio; se obtuvo su consentimiento, pero no se les informó cuál era el tiempo ideal de uso de pantallas. Luego se les solicitó a las madres que rellenaran una encuesta estructurada y la ERC. Las encuestas y las escalas se recolectaron durante la consulta. Se proporcionó asesoramiento para los puntos en los que se detectaron problemas y se planificó una supervisión.

\section{Encuesta estructurada}

La encuesta estructurada en papel contaba con 24 preguntas que debían responder las madres. La edad y el sexo del niño, la edad y el nivel educativo de los padres, la condición laboral de la madre, la estructura familiar, la cantidad de hijos en la familia, el tipo de familia, el cuidador del niño (la madre u otra persona) y el nivel de ingresos formaron parte de las preguntas relativas a los datos sociodemográficos. En función del uso diario de pantallas, se dividió a los participantes en uso bajo ( $\leq 1 \mathrm{~h} /$ día) o alto ( $\geq 4$ h/ día). También se indagó acerca de la edad que tenía el niño la primera vez que estuvo expuesto a una pantalla, si los padres lo acompañaban al usar pantallas, si reaccionaba ante las limitaciones del tiempo de uso de pantallas y si posponía las necesidades diarias.

\section{Emotion Regulation Checklist (ERC)}

La ERC fue desarrollada en 1997 por Shields y Cicchetti. ${ }^{15}$ La validez, la fiabilidad y la adaptación de la escala para niños turcos fueron estudiadas por Batum en 2007. ${ }^{16}$ La ERC puede ser respondida por adultos que conocen bien al niño, como el padre, la madre o el cuidador. La ERC tiene 24 ítems que se evalúan en una escala Likert de 4 puntos ( 1 = nunca; 2 = alguna vez; 3 = frecuentemente; 4 = casi siempre). La ERC incluye dos subescalas: la subescala de labilidad/ negatividad (S-L/N) y la subescala de regulación emocional (S-RE). La S-RE está compuesta por 10 ítems que pretenden determinar la autoconciencia emocional y la expresión emocional constructiva del niño. La S-L/ N está compuesta por 13 ítems que evalúan la falta de flexibilidad, la activación emocional, la reactividad, la desregulación de la ira y la labilidad emocional. Se determinó que la coherencia interna de ambas subescalas es adecuada (L/N: $\alpha=0,96$; RE: $\alpha=0,83$ ). Una puntuación alta en la escala indica que el niño tiene un gran nivel de habilidades de regulación emocional.

\section{Análisis estadístico}

Las estadísticas descriptivas se presentaron como números y porcentajes para los datos categóricos. La prueba de $\chi^{2}$ se utilizó para comparar las frecuencias de las variables categóricas. Se realizó un análisis multivariado de regresión logística para determinar qué factores están asociados con el tiempo de uso de pantallas por parte de los niños luego de ajustar en función del sexo, la educación materna, la educación paterna, la ocupación de la madre, el cuidador, la cantidad de hijos, el orden de nacimiento, la edad durante la primera exposición a una pantalla y ver pantallas en compañía de los padres. Las razones de posibilidades ajustadas se calcularon con intervalos de confianza del $95 \%$. La puntuación total de ERC, la puntuación de RE y la puntuación de L / N se dividieron en cuartiles, y la proporción de participantes con los cuartiles más altos se utilizó para la comparación. El nivel de significancia estadística se estableció en $p<0,05$. El análisis estadístico se realizó con el programa SPSS, versión 22.0 (IBM Corp., Armonk, NY, EE. UU.).

\section{RESULTADOS}

De los 240 niños que participaron en el estudio, en 98 (el 40,8\%) se observó un uso de pantallas $\geq 4$ horas. En la Tabla 1, se muestran las asociaciones entre los factores sociodemográficos y el tiempo de uso de pantallas. En la Tabla 2, se observa el análisis de regresión logística de los factores asociados con el tiempo de uso de pantallas en los niños. Ser cuidado por la madre, tener 12 meses o más durante la primera exposición a pantallas y no estar acompañado por los padres al usar pantallas se asociaron con $\geq 4$ horas de uso de pantallas.

En la Tabla 3, se muestran las asociaciones entre las características sociodemográficas y los participantes con puntuaciones altas de ERC. En la Tabla 4, se muestran las asociaciones entre las características del tiempo de uso de pantallas y las puntuaciones altas de ERC. La proporción de participantes con una puntuación alta de L/N 
TABLA 1. Relaciones entre los factores sociodemográficos y el tiempo de uso de pantallas en los niños*

\begin{tabular}{|c|c|c|c|c|}
\hline & & \multicolumn{2}{|c|}{ Tiempo de uso de pantallas } & \multirow[b]{2}{*}{$p$} \\
\hline & & $\begin{array}{c}\leq 1 \text { hora } \\
\mathrm{n}=142(58,2 \%)\end{array}$ & $\begin{array}{c}\geq 4 \text { horas } \\
\mathrm{n}=98(40,8 \%)\end{array}$ & \\
\hline Edad & $\begin{array}{l}<48 \text { meses } \\
\geq 48 \text { meses }\end{array}$ & $\begin{array}{l}95(63,3 \%) \\
47(52,2 \%)\end{array}$ & $\begin{array}{l}55(36,7 \%) \\
43(47,8 \%)\end{array}$ & 0,090 \\
\hline Sexo & $\begin{array}{l}\text { Femenino } \\
\text { Masculino }\end{array}$ & $\begin{array}{l}78(66,1 \%) \\
64(52,5 \%)\end{array}$ & $\begin{array}{l}40(33,9 \%) \\
58(47,5 \%)\end{array}$ & 0,032 \\
\hline Edad materna, media $\pm \mathrm{DE}$ & Años & $31,8 \pm 5,0$ & $32,1 \pm 5,3$ & 0,865 \\
\hline Edad paterna, media $\pm \mathrm{DE}$ & Años & $34,2 \pm 4,8$ & $35,7 \pm 6,0$ & 0,214 \\
\hline Educación materna & $\begin{array}{l}\text { Escuela secundaria o inferior } \\
\text { Superior a la escuela secundaria }\end{array}$ & $\begin{array}{l}58(46,8 \%) \\
84(72,4 \%)\end{array}$ & $\begin{array}{l}66(53,2 \%) \\
32(27,6 \%)\end{array}$ & $<0,001$ \\
\hline Educación paterna & $\begin{array}{l}\text { Escuela secundaria o inferior } \\
\text { Superior a la escuela secundaria }\end{array}$ & $\begin{array}{l}63(51,6 \%) \\
79(66,9 \%)\end{array}$ & $\begin{array}{l}59(48,4 \%) \\
39(33,1 \%)\end{array}$ & 0,016 \\
\hline Ocupación de la madre & $\begin{array}{l}\text { Ama de casa } \\
\text { Trabaja fuera de la casa }\end{array}$ & $\begin{array}{l}61(50,0 \%) \\
81(68,6 \%)\end{array}$ & $\begin{array}{l}61(50,0 \%) \\
37(31,4 \%)\end{array}$ & 0,003 \\
\hline Cuidador & $\begin{array}{l}\text { Madre } \\
\text { Abuelos/niñera } \\
\text { Jardín de infantes }\end{array}$ & $\begin{array}{l}58(46,8 \%) \\
45(66,2 \%) \\
39(81,3 \%)\end{array}$ & $\begin{array}{c}66(53,2 \%) \\
23(33,8 \%) \\
9(18,8 \%)\end{array}$ & $<0,001$ \\
\hline Cantidad de hijos & $\begin{array}{l}1 \\
\geq 2\end{array}$ & $\begin{array}{l}65(71,4 \%) \\
77(51,7 \%)\end{array}$ & $\begin{array}{l}26(28,6 \%) \\
72(48,3 \%)\end{array}$ & 0,003 \\
\hline Orden de nacimiento & $\begin{array}{l}1 \\
\geq 2\end{array}$ & $\begin{array}{l}88(66,7 \%) \\
54(50,0 \%)\end{array}$ & $\begin{array}{l}44(33,3 \%) \\
54(50,0 \%)\end{array}$ & 0,009 \\
\hline Edad durante la $1^{\text {a }}$ exposición a pantallas & $\begin{array}{l}<12 \text { meses } \\
\geq 12 \text { meses }\end{array}$ & $\begin{array}{c}39(45,3 \%) \\
103(66,9 \%)\end{array}$ & $\begin{array}{l}47(54,7 \%) \\
51(33,1 \%)\end{array}$ & 0,001 \\
\hline Uso de pantallas con los padres & $\begin{array}{l}\text { Sí } \\
\text { No }\end{array}$ & $\begin{array}{l}114(64,8 \%) \\
28(43,8 \%)\end{array}$ & $\begin{array}{l}62(35,2 \%) \\
36(56,3 \%)\end{array}$ & 0,003 \\
\hline
\end{tabular}

${ }^{*} \mathrm{n}(\%) /$ media $\pm \mathrm{DE}$

TABLa 2. Análisis de regresión logística de los factores asociados con el tiempo de uso de pantallas en los niños*

\begin{tabular}{|c|c|c|c|c|c|}
\hline & \multicolumn{2}{|c|}{ Tiempo de uso de pantallas ( $\geq 4$ horas) } & \multicolumn{2}{|c|}{ IC del $95 \%^{b}$} & \multirow[b]{2}{*}{$p$} \\
\hline & & $A O R^{\mathrm{a}}$ & Inferior & Superior & \\
\hline Sexo & $\begin{array}{l}\text { Femenino } \\
\text { Masculino }\end{array}$ & $\begin{array}{c}0,574 \\
1\end{array}$ & 0,32 & 1,04 & 0,067 \\
\hline Educación materna & $\begin{array}{l}\text { Escuela secundaria o inferior } \\
\text { Superior a la escuela secundaria }\end{array}$ & $\begin{array}{c}1,67 \\
1\end{array}$ & 0,74 & 3,84 & 0,217 \\
\hline Educación paterna & $\begin{array}{l}\text { Escuela secundaria o inferior } \\
\text { Superior a la escuela secundaria }\end{array}$ & $\begin{array}{c}2,01 \\
1\end{array}$ & 0,87 & 4,62 & 0,101 \\
\hline Ocupación de la madre & $\begin{array}{l}\text { Ama de casa } \\
\text { Trabaja fuera de la casa }\end{array}$ & $\begin{array}{c}4,84 \\
1\end{array}$ & 0,87 & 26,91 & 0,071 \\
\hline Cuidador & $\begin{array}{l}\text { Madre } \\
\text { Abuelos/niñera } \\
\text { Jardín de infantes }\end{array}$ & $\begin{array}{c}16,88 \\
2,05 \\
1\end{array}$ & $\begin{array}{l}2,85 \\
0,81\end{array}$ & $\begin{array}{c}99,96 \\
5,19\end{array}$ & $\begin{array}{l}0,002 \\
0,129\end{array}$ \\
\hline Cantidad de hijos & $\begin{array}{l}1 \\
\geq 2\end{array}$ & $\begin{array}{c}1,08 \\
1\end{array}$ & 0,43 & 2,71 & 0,866 \\
\hline Orden de nacimiento & $\begin{array}{l}1 \\
\geq 2\end{array}$ & $\begin{array}{c}0,52 \\
1\end{array}$ & 0,22 & 1,21 & 0,130 \\
\hline Edad durante la $1^{\text {a }}$ exposición a pantallas & $\begin{array}{l}<12 \text { meses } \\
\geq 12 \text { meses }\end{array}$ & $\begin{array}{c}2,62 \\
1\end{array}$ & 1,43 & 4,80 & 0,002 \\
\hline Uso de pantallas con los padres & $\begin{array}{l}\text { Sí } \\
\text { No }\end{array}$ & $\begin{array}{c}0,42 \\
1\end{array}$ & 0,21 & 0,82 & 0,012 \\
\hline
\end{tabular}

* Ajustado en función del sexo, la educación materna, la educación paterna, la ocupación de la madre, el cuidador, la cantidad de hijos, el orden de nacimiento, la edad durante la primera exposición a una pantalla y ver pantallas en compañía de los padres.

** Categoría de referencia: $\leq 1$ hora.

a Razón de posibilidades ajustada.

b Intervalo de confianza. 
fue significativamente mayor entre los niños con un uso de pantallas $\geq 4$ horas y que no estaban acompañados por sus padres al usar pantallas $(p=0,004 ; p=0,033$, respectivamente).

\section{DISCUSIÓN}

Los avances en las tecnologías de la información y la comunicación han incrementado el uso de los dispositivos tecnológicos. Como consecuencia de este aumento en el uso, a los niños preescolares les resulta más fácil familiarizarse con dispositivos como tabletas y teléfonos inteligentes antes de adquirir habilidades básicas. ${ }^{17}$ Con el objetivo de revelar los posibles efectos de la exposición a pantallas, en este estudio se compararon los grupos con

TABLA 3. Asociación entre las características sociodemográficas y la proporción de cuartiles más altos de puntuaciones en la escala de regulación emocional*

\begin{tabular}{|c|c|c|c|c|c|c|c|c|}
\hline & & $\begin{array}{c}\text { Total } \\
\mathbf{n}\end{array}$ & $\begin{array}{l}\text { Puntuación } \\
\text { de RE } \\
4^{\circ} \text { cuartil }\end{array}$ & $p$ & $\begin{array}{c}\text { Puntuación } \\
\text { de } \mathrm{L} / \mathrm{N} \\
4^{\circ} \text { cuartil }\end{array}$ & $p$ & $\begin{array}{c}\text { Puntuación } \\
\text { total } \\
4^{\circ} \text { cuartil }\end{array}$ & $p$ \\
\hline Edad & $\begin{array}{l}<48 \text { meses } \\
\geq 48 \text { meses }\end{array}$ & $\begin{array}{c}150 \\
90\end{array}$ & $\begin{array}{l}50(33,3 \%) \\
25(27,8 \%)\end{array}$ & 0,369 & $\begin{array}{l}38(25,3 \%) \\
31(34,4 \%)\end{array}$ & 0,131 & $\begin{array}{l}45(30,0 \%) \\
18(20,0 \%)\end{array}$ & 0,088 \\
\hline Sexo & $\begin{array}{l}\text { Femenino } \\
\text { Masculino }\end{array}$ & $\begin{array}{l}118 \\
122\end{array}$ & $\begin{array}{l}41(34,7 \%) \\
34(27,9 \%)\end{array}$ & 0,251 & $\begin{array}{l}29(24,6 \%) \\
40(32,8 \%)\end{array}$ & 0,160 & $\begin{array}{l}34(28,8 \%) \\
29(23,8 \%)\end{array}$ & 0,375 \\
\hline Edad materna & $\begin{array}{l}<30 \text { años } \\
\geq 30 \text { años }\end{array}$ & $\begin{array}{l}103 \\
137\end{array}$ & $\begin{array}{l}32(31,1 \%) \\
43(31,4 \%)\end{array}$ & 0,958 & $\begin{array}{l}29(28,2 \%) \\
40(29,2 \%)\end{array}$ & 0,860 & $\begin{array}{l}25(24,3 \%) \\
38(27,7 \%)\end{array}$ & 0,546 \\
\hline Edad paterna & $\begin{array}{l}<30 \text { años } \\
\geq 30 \text { años }\end{array}$ & $\begin{array}{c}53 \\
187\end{array}$ & $\begin{array}{l}20(37,7 \%) \\
55(29,4 \%)\end{array}$ & 0,248 & $\begin{array}{l}14(26,4 \%) \\
55(29,4 \%)\end{array}$ & 0,670 & $\begin{array}{l}13(24,5 \%) \\
50(26,7 \%)\end{array}$ & 0,747 \\
\hline Educación materna & $\begin{array}{l}\text { Escuela secundaria o inferior } \\
\text { Superior a la escuela secundaria }\end{array}$ & $\begin{array}{c}67 \\
173\end{array}$ & $\begin{array}{l}25(37,3 \%) \\
50(28,9 \%)\end{array}$ & 0,207 & $\begin{array}{l}16(23,9 \%) \\
53(30,6 \%)\end{array}$ & 0,300 & $\begin{array}{l}21(31,3 \%) \\
42(24,3 \%)\end{array}$ & 0,264 \\
\hline Educación paterna & $\begin{array}{l}\text { Escuela secundaria o inferior } \\
\text { Superior a la escuela secundaria }\end{array}$ & $\begin{array}{c}58 \\
152\end{array}$ & $\begin{array}{l}22(37,9 \%) \\
53(29,1 \%)\end{array}$ & 0,207 & $\begin{array}{l}19(32,8 \%) \\
50(27,5 \%)\end{array}$ & 0,439 & $\begin{array}{l}14(24,1 \%) \\
49(26,9 \%)\end{array}$ & 0,675 \\
\hline Ocupación de la madre & $\begin{array}{l}\text { Ama de casa } \\
\text { Trabaja fuera de la casa }\end{array}$ & $\begin{array}{l}122 \\
118\end{array}$ & $\begin{array}{l}36(29,5 \%) \\
39(33,1 \%)\end{array}$ & 0,554 & $\begin{array}{l}36(29,5 \%) \\
33(28,0 \%)\end{array}$ & 0,792 & $\begin{array}{l}32(26,2 \%) \\
31(26,3 \%)\end{array}$ & 0,994 \\
\hline Cuidador & $\begin{array}{l}\text { Madre } \\
\text { Abuelos/niñera } \\
\text { Jardín de infantes }\end{array}$ & $\begin{array}{c}125 \\
65 \\
50\end{array}$ & $\begin{array}{l}36(28,8 \%) \\
25(38,5 \%) \\
14(28,0 \%)\end{array}$ & 0,338 & $\begin{array}{l}40(32,0 \%) \\
16(24,6 \%) \\
10(26,0 \%)\end{array}$ & 0,504 & $\begin{array}{l}31(24,8 \%) \\
18(27,7 \%) \\
14(28,0 \%)\end{array}$ & 0,867 \\
\hline Cantidad de hijos & $\begin{array}{l}1 \\
\geq 2\end{array}$ & $\begin{array}{c}91 \\
149\end{array}$ & $\begin{array}{l}24(26,4 \%) \\
51(34,2 \%)\end{array}$ & 0,203 & $\begin{array}{l}22(24,2 \%) \\
47(31,5 \%)\end{array}$ & 0,221 & $\begin{array}{l}23(25,3 \%) \\
40(26,8 \%)\end{array}$ & 0,788 \\
\hline Orden de nacimiento & $\begin{array}{l}1 \\
\geq 2\end{array}$ & $\begin{array}{l}132 \\
108\end{array}$ & $\begin{array}{l}39(29,5 \%) \\
36(33,3 \%)\end{array}$ & 0,529 & $\begin{array}{l}37(28,0 \%) \\
32(29,6 \%)\end{array}$ & 0,785 & $\begin{array}{l}31(23,5 \%) \\
32(29,6 \%)\end{array}$ & 0,282 \\
\hline
\end{tabular}

${ }^{*} \mathrm{n}(\%)$.

Tabla 4. Asociación entre las características del tiempo de uso de pantallas y la proporción de cuartiles más altos de puntuaciones en la escala de regulación emocional*

\begin{tabular}{|c|c|c|c|c|c|c|c|c|}
\hline & & $\begin{array}{c}\text { Total } \\
\mathbf{n}\end{array}$ & $\begin{array}{c}\text { Puntuación } \\
\text { de RE } \\
4 .^{\circ} \text { cuartil }\end{array}$ & $p$ & $\begin{array}{l}\text { Puntuación } \\
\text { de } \mathrm{L} / \mathrm{N} \\
4 .^{\circ} \text { cuartil }\end{array}$ & $p$ & $\begin{array}{c}\text { Puntuación } \\
\text { total } \\
4 .^{\circ} \text { cuartil }\end{array}$ & $p$ \\
\hline Tiempo de uso de pantallas & $\begin{array}{l}<1 \text { hora } \\
\geq 4 \text { horas }\end{array}$ & $\begin{array}{c}142 \\
98\end{array}$ & $\begin{array}{l}50(35,2 \%) \\
25(25,5 \%)\end{array}$ & 0,111 & $\begin{array}{l}31(21,8 \%) \\
38(38,8 \%)\end{array}$ & 0,004 & $\begin{array}{l}37(26,1 \%) \\
26(26,5 \%)\end{array}$ & 0,935 \\
\hline $\begin{array}{l}\text { Edad durante la } 1^{\mathrm{a}} \text { exposición } \\
\text { a pantallas }\end{array}$ & $\begin{array}{l}<12 \text { meses } \\
\geq 12 \text { meses }\end{array}$ & $\begin{array}{c}86 \\
154\end{array}$ & $\begin{array}{l}25(29,1 \%) \\
50(32,5 \%)\end{array}$ & 0,586 & $\begin{array}{l}28(32,6 \%) \\
41(26,6 \%)\end{array}$ & 0,330 & $\begin{array}{l}23(26,7 \%) \\
40(26,0 \%)\end{array}$ & 0,897 \\
\hline $\begin{array}{l}\text { Uso de pantallas } \\
\text { con los padres }\end{array}$ & $\begin{array}{l}\text { Sí } \\
\text { No }\end{array}$ & $\begin{array}{c}176 \\
64\end{array}$ & $\begin{array}{l}61(34,7 \%) \\
14(21,9 \%)\end{array}$ & 0,059 & $\begin{array}{l}44(25,0 \%) \\
25(39,1 \%)\end{array}$ & 0,033 & $\begin{array}{l}49(27,8 \%) \\
14(21,9 \%)\end{array}$ & 0,353 \\
\hline $\begin{array}{l}\text { Reacciona ante las limitaciones } \\
\text { del tiempo de uso de pantallas }\end{array}$ & $\begin{array}{l}\text { Sí } \\
\text { No }\end{array}$ & $\begin{array}{c}93 \\
147\end{array}$ & $\begin{array}{l}25(26,9 \%) \\
50(34,0 \%)\end{array}$ & 0,246 & $\begin{array}{l}31(33,3 \%) \\
38(25,9 \%)\end{array}$ & 0,212 & $\begin{array}{l}22(23,7 \%) \\
41(27,9 \%)\end{array}$ & 0,468 \\
\hline Pospone las necesidades diarias & $\begin{array}{l}\text { Sí } \\
\text { No }\end{array}$ & $\begin{array}{l}121 \\
119\end{array}$ & $\begin{array}{l}35(28,9 \%) \\
40(33,6 \%)\end{array}$ & 0,433 & $\begin{array}{l}39(32,2 \%) \\
30(25,2 \%)\end{array}$ & 0,230 & $\begin{array}{l}29(24,0 \%) \\
34(29,6 \%)\end{array}$ & 0,418 \\
\hline
\end{tabular}


el menor tiempo de uso de pantallas y aquellos que superaban ampliamente el tiempo de uso de pantallas recomendado.

Es posible que pasar tiempo usando pantallas e interactuando con ellas en lugar de con los padres $u$ otros seres humanos reduzca el tiempo en que los niños pueden practicar sus habilidades para reconocer las emociones de los demás y experimentar las emociones suscitadas al interactuar con otros. Skalická y cols., ${ }^{18}$ sugirieron que la exposición prolongada a pantallas podría afectar los procesos de socialización de los niños pequeños y derivar en una menor capacidad para comprender las emociones. La RE se plantea como la capacidad de modulación emocional que permite que las personas establezcan la relación más adecuada con el entorno. Es fundamental para iniciar, motivar y organizar la conducta adaptativa y necesaria para prevenir niveles estresantes de emociones negativas. La labilidad emocional/negatividad se describe como la rapidez que tiene un niño para responder a emociones que provocan estímulos y la dificultad simultánea para recuperarse de reacciones emocionales negativas. ${ }^{19}$ Se informó que los niños con una mayor labilidad emocional/ negatividad tenían un menor funcionamiento social adecuado. ${ }^{20}$ Además, los niños con una elevada emotividad negativa, como la ira, eran más propensos a tener problemas interiorizados y exteriorizados durante la niñez y la adolescencia. ${ }^{21}$

Dado que se trata de un factor presente en la vida de los niños que compite con el comportamiento de los padres y cuyos efectos aumentan día a día, la pregunta es la siguiente: "¿Hasta qué punto el tiempo de uso de pantallas afecta la RE?". El uso excesivo de pantallas (p. ej., más de 2 a 3 h de exposición a medios electrónicos) puede afectar el cerebro en desarrollo, con importantes consecuencias en el desarrollo cognitivo y motriz, el aprendizaje y la memoria, la RE y la salud en general. En las investigaciones se sugiere que la exposición temprana y prolongada a medios electrónicos está asociada con un mayor riesgo de tener síntomas psiquiátricos, en especial problemas de atención e hiperactividad, trastornos de ansiedad y depresión. ${ }^{22}$ También se demostró que una elevada exposición a la televisión de fondo en menores de 5 años reduce la cantidad y la calidad de las interacciones entre padres e hijos y les quita tiempo de juego, afecta negativamente el uso y la adquisición del lenguaje, la atención, el desarrollo cognitivo y la función ejecutiva. ${ }^{23}$
En la bibliografía existente relativa a niños más grandes y adolescentes, hay posturas divididas sobre la relación entre el uso de pantallas y el bienestar: en algunos estudios se observaron efectos perjudiciales y en otros, correlaciones de daño desdeñables y no significativas. Hay poco conocimiento sobre cómo el tiempo de uso de pantallas se vincula con el bienestar psicológico, en especial en menores de 5 años. ${ }^{24}$ En un estudio reciente, se observó que los niños y adolescentes de 2 a 17 años que pasaban más tiempo delante de una pantalla tenían menor bienestar psicológico que aquellos que pasaban menos tiempo. Se observó que los usuarios intensivos de las pantallas eran significativamente más propensos a tener una regulación emocional deficiente, que implicaba perder la calma, discutir en exceso y tener dificultades para llevarse bien con otras personas. ${ }^{25}$ En este estudio, también se observó que los niños con un uso excesivo de pantallas eran más inestables y negativos emocionalmente. Además, no se halló una relación entre el componente de la RE y el tiempo de uso de pantallas. La Academia Estadounidense de Pediatría también recomienda que los padres acompañen a sus hijos mientras usan pantallas y los ayuden a comprender lo que están viendo. ${ }^{3} \mathrm{En}$ este estudio, se logró justificar esta propuesta en relación con la regulación emocional, ya que se determinó que los niños que estaban solos delante de pantallas eran más inestables emocionalmente. Por lo tanto, dado que muchas madres no tienen suficientes conocimientos sobre el uso adecuado de pantallas en sus hijos, como el uso acompañado por los padres, es importante indagar sobre los hábitos de uso de los medios e informarlas del uso correcto. Durante la pandemia, el tiempo de uso de pantallas entre los niños se vio afectado. Incluso en estas condiciones, se debe hacer hincapié en la importancia de la vida familiar y la actividad física para los niños. En estudios anteriores se informaron resultados discordantes sobre la relación existente entre la educación materna, la educación paterna, el sexo del niño y el tiempo de uso de pantallas. ${ }^{26,27}$ De forma similar a los resultados expuestos por Trinh y cols., ${ }^{28}$ en este estudio se demostró una relación inversa entre el nivel de educación de los padres y el tiempo de uso de pantallas en los niños, y también se observó que el sexo masculino estaba asociado con un mayor tiempo de uso de pantallas. Además, Maatta y cols., ${ }^{29}$ demostraron que los padres con un mayor nivel de educación imponían menos normas descriptivas, usaban 
menos pantallas delante de sus hijos y le daban más importancia a limitar el tiempo de uso de pantallas en los niños en comparación con los padres con un nivel educativo menor.

Los hallazgos relacionados con la ocupación de la madre, la presencia de hermanos y el tiempo de uso de pantallas en los niños difirieron en estudios anteriores. ${ }^{28}$ Hinkley y cols. ${ }^{30}$ no hallaron ninguna asociación entre el trabajo de la madre, la presencia de hermanos y el tiempo de uso de pantallas en niños preescolares. Por el contrario, $\mathrm{Wu}$ y cols. ${ }^{31}$ informaron que los niños cuyas madres eran amas de casa usaban dispositivos electrónicos con mayor frecuencia que aquellos cuyas madres trabajaban fuera del hogar. De conformidad con estos hallazgos, en un estudio anterior, se observó que los niños que recibían cuidado en sus hogares eran más propensos a estar expuestos a la televisión que aquellos que acudían a un jardín de infantes. ${ }^{32}$ En este estudio, se observó que el hecho de que la madre fuera ama de casa y la presencia de hermanos eran determinantes positivos para el tiempo de uso de pantallas. También se observó que el tiempo de uso de pantallas de los niños que acudían a un jardín de infantes era significativamente menor. Es posible que la presencia de hermanos aumente la carga de trabajo de la madre y disminuya el tiempo de calidad que el niño pasa con ella, por lo que el tiempo de uso de pantallas aumenta cuando los niños son cuidados en sus hogares. Otro posible motivo es que los hermanos son modelos en lo que respecta al uso de las pantallas.

La fortaleza de este estudio es que es el primero en examinar la asociación entre la RE y el uso excesivo de pantallas en niños preescolares con una escala estandarizada. En este estudio, también hay algunas limitaciones que deben tenerse en cuenta. En primer lugar, las madres informaron el tiempo de uso de pantallas, por lo que es posible que se lo haya subestimado. No obstante, se espera que las madres tengan una noción acerca del tiempo ideal de uso de pantallas para tomar partido al responder el cuestionario. En este estudio, es posible considerar que la probabilidad de sesgo disminuye por la gran proporción de madres que informaron que sus hijos pasan más de 4 horas delante de pantallas. En segundo lugar, si bien las madres participantes no tenían un diagnóstico de enfermedades psiquiátricas, su bienestar psicológico puede constituir un factor de confusión. Esto debería tenerse en cuenta en estudios futuros. En tercer lugar, no se indagó sobre la cantidad total de horas que los niños permanecían en el jardín de infantes. No obstante, independientemente de cuánto permanecían en el jardín de infantes, el hecho de que los niños acudieran a uno se asoció con poco tiempo de uso de pantallas.

\section{CONCLUSIONES}

En este estudio, donde se investigó la relación entre las habilidades de regulación emocional y el uso de pantallas, se observó que un uso excesivo de pantallas se asocia con labilidad emocional durante esta etapa temprana de la infancia.

\section{REFERENCIAS}

1. Sevcikova A. Two sides of the same coin: communication technology, media use, and our kids' health. Int J Public Health. 2015; 60(2):129-30.

2. Radesky JS, Schumacher J, Zuckerman B. Mobile and interactive media use by young children: the good, the bad, and the unknown. Pediatrics. 2015; 135(1):1-3.

3. Council on Communications and Media. Media and young minds. Pediatrics. 2016; 138(5):e20162591.

4. American Academy of Child \& Adolescent Psychiatry. Media Habits During COVID-19: Children \& Teens on Screens in Quarantine. AACAP. 2020. [Fecha de acceso: 14 de septiembrede2020]. Disponibleen: https: / / www.aacap. org / App_Themes / AACAP / Docs / resource_libraries / covid-19/Screen-Time-During-COVID.pdf

5. Kostyrka-AllchorneK, CooperNR,SimpsonA. Therelationship between television exposure and children's cognition and behaviour: A systematic review. Dev Rev. 2017; 44:19-58.

6. Lissak G. Adverse physiological and psychological effects of screen time on children and adolescents: literature review and case study. Environ Res. 2018; 164:149-57.

7. Schmidt ME, Rich M, Rifas-Shiman SL, Oken E, et al. Television viewing in infancy and child cognition at 3 years of age in a US cohort. Pediatrics. 2009; 123(3):e370-5.

8. Linebarger DL, Vaala SE. Screen media and language development in infants and toddlers: An ecological perspective. Dev Rev. 2010; 30(2):176-202.

9. Thompson R. Emotion regulation: a theme in search of a definition. En: Fox NA, ed. Emotion Regulation: Biological and Behavioral Considerations. Monographs of the Society for Research in Child Development. Chicago: University of Chicago Press; 1994; 59:25-52.

10. Roque L, Verissimo M, Fernandes M, Rebelo A. Emotion regulation and attachment: Relationships with children's secure base, during different situational and social contexts in naturalistic settings. Infant Behav Dev. 2013; 36(3):298306.

11. Garber J, Dodge KA. Cambridge studies in social and emotional development. The development of emotion regulation and dysregulation. Cambridge: University Press; 1991.

12. Akçay D, ÖzcebeH. Evaluation of Computer Game Playing Habits of Children at Pre-School Education Levels and Their Families. J Child. 2012; 12:66-71.

13. YalçınS, Yıldız YahşiD, Oflu A, TezolÖ, at al. Multi-centered investigation of pre-school children's screen time and digital game playing habits. Fulltext paper. International Child and Information Safety Congress "Digital Games". April 11-13, 2018. Ankara.

14. Waisman I, Hidalgo E, Rossi ML. Uso de pantallas en niños pequeños en una ciudad de Argentina. Arch Argent Pediatr. 2018; 116(2):186-95. 
15. Shields A, CicchettiD. Emotion regulation among school-age children: The development and validation of a new criterion Q-sort scale. Dev Psychol. 1997; 33(6):906-16.

16. Batum P, Yağmurlu B. What counts in externalizing behaviors? The contributions of emotion and behavior regulation. Curr Psychol. 2007; 25:272-94.

17. Ergüney M. İnternetin Okul Öncesi Dönemdeki Çocuklar Üzerindeki Etkileri Hakkında Bir Araştırma. Ulakbilge. 2017; 5(17):1917-38.

18. Skalická V, Wold Hygen B, Stenseng F, Kårstad SB, et al. Screen time and the development of emotion understanding from age 4 to age 8: A community study. Br J Dev Psychol. 2019; 37:427-43.

19. Kim-Spoon J, Cicchetti D, Rogosch FA. A longitudinal study of emotion regulation, emotion lability-negativity, and internalizing symptomatology in maltreated and nonmaltreated children. Child Dev. 2013; 84(2):512-27.

20. Eisenberg N, Fabes RA, Murphy B, MaszkP, etal. The role of emotionality and regulation in children's social functioning: A longitudinal study. Child Dev. 1995; 66(5):1360-84.

21. Kim J, Deater-Deckard K. Dynamic changes in anger linking to developmental trajectories of internalizing and externalizing problems: The moderating role of attention. J Child Psychol Psychiatry. 2011; 52(2):156-66.

22. Neophytou E, Manwell L, Eikelboom R. Effects of Excessive Screen Time on Neurodevelopment, Learning, Memory, Mental Health, and Neurodegeneration: a Scoping Review. Int J Ment Health Addict. 2019; [En prensa].

23. Canadian Paediatric Society, Digital Health Task Force, Ottawa, Ontario. Screen time and young children: Promoting health and development in a digital world.
Paediatr Child Health. 2018; 23(1):83.

24. Przybylski AK, Weinstein N. Digital screen time limits and young children's psychological well-being: Evidence from a population-based study. Child Dev. 2019; 90(1):e56-65.

25. Twenge JM, Campbell WK. Associations between screen time and lower psychological well-being among children and adolescents: Evidence from a population-based study. Prev Med Rep. 2018; 12:271-83.

26. Duch H, Fisher EM, Ensari I, Harrington A. Screen time use in children under 3 years old: a systematic review of correlates. Int J Behav Nutr Phys Act. 2013; 10:102.

27. Carson V, Janssen I. Associations between factors within the home setting and screen time among children aged 0-5 years: a cross-sectional study. BMC Public Health. 2012; 12:539.

28. Trinh $\mathrm{MH}$, Sundaram R, Robinson SL, Lin TC, et al. Association of Trajectory and Covariates of Children's Screen Media Time. JAMA Pediatr. 2019; 174(1):71-8.

29. Määttä S, Kaukonen R, Vepsäläinen H, Lehto E, et al. The mediating role of the home environment in relation to parental educational level and preschool children's screen time: a cross-sectional study. BMC Public Health. 2017; 17(1):688.

30. Hinkley T, Salmon J, Okely AD, Trost SG. Correlates of sedentary behaviours in preschool children: a review. Int J Behav Nutr Phys Act. 2010; 7:66.

31. Wu CST, Fowler C, Lam WYY, Wong HT, et al. Parenting approaches and digital technology use of preschool age children in a Chinese community. Ital J Pediatr. 2014; 40:44.

32. Christakis DA, Garrison MM. Preschool-aged children's television viewing in child care settings. Pediatrics. 2009; 124(6):1627-32.

\section{Artículos seleccionados}

Los siguientes resúmenes y comentarios de trabajos seleccionados se encuentran disponibles en la versión electrónica de este número.

Arch Dis Child Fetal Neonatal Ed. 2020 Sep 28:fetalneonatal-2020-319767.

Incidencia de retinopatía del prematuro en Alemania: evaluación de los criterios actuales de detección (Larsen PP, et al. Incidence of retinopathy of prematurity in Germany: evaluation of current detection criteria) Comentario: Dr. José María Ceriani Cernadas. Editor Archivos Argentinos de Pediatría.

BMJ. 2020 Nov 25;371:m4075.

Edad gestacional e ingresos hospitalarios durante la infancia: estudio con base poblacional de vinculación de registros en Inglaterra (estudio TIGAR) (Coathup V, et al. Gestational age and hospital admissions during childhood: population based, record linkage study in England (TIGAR study))

Comentario: Dr. Néstor E. Vain. Sanatorios de la Trinidad Palermo, San Isidro y Ramos Mejía.

Dra. Fabiana Herbón. Sanatorios de la Trinidad Palermo y Ramos Mejía.

JAMA Pediatr. 2020 Sep 28;174(11):1-7.

Introducción temprana de gluten y enfermedad celíaca en el estudio EAT: un análisis preespecificado del estudio clínico aleatorizado EAT (Logan K, et al. Early gluten introduction and celiac disease in the eat study: a prespecified analysis of the eat randomized clinical trial)

Comentario: Dra. María del Carmen Toca. Hospital Nacional Alejandro Posadas. Buenos Aires. 